\title{
Diagnostic utility of PET/CT in a patient with miliary tuberculosis
}

\author{
Cuneyt Tetikkurt ${ }^{1}$, Nurbanu Inci ${ }^{2}$, Halil Yanardag² \\ ${ }^{1}$ Pulmonary Diseases Department, Cerrahpasa Medical Faculty, Istanbul Cerrahpasa University; ${ }^{2}$ Internal Medicine \\ Department, Cerrahpasa Medical Faculty, Istanbul Cerrahpasa University, Turkey
}

\begin{abstract}
A 63-year-old male presented with loss of appetite, subfebrile fever, swelling of the right hand and dyspnea on exertion for three months. Past medical history revealed methotrexate treatment of six months for rheumatoid arthritis. Chest radiography and computed tomography $(\mathrm{CT})$ revealed diffuse miliary nodules. PET/CT scan demonstrated diffuse FDG uptake in both lungs, in the spleen, in the right hand, the mediastinal and the axillary lymph nodes. MR of the right hand showed inflammatory arthritis. Histopathology of the right hand tru-cut biopsy revealed degenerative changes. Culture of the hand biopsy tissue was positive for mycobacterium tuberculosis.

PET/CT may determine the biopsy and the sampling sites for the early diagnosis of patients with suspected miliary tuberculosis where lesion identification on other modalities may be difficult or unfeasible. High sensitivity for inflammatory diseases makes PET/CT a useful diagnostic utility for enabling early diagnosis in miliary tuberculosis which is a diagnostic predicament.
\end{abstract}

Correspondence: Cuneyt Tetikkurt, Pulmonary Diseases Department, Cerrahpasa Medical Faculty, Istanbul Cerrahpasa University, Tanzimat sokak Serkan Apartmanı, No 8/16, Caddebostan 34728, Istanbul, Turkey.

Tel. +90.532 .3810900 - Fax: +90.212 .5870217 .

E-mail: tetikkurt@gmail.com

Contributions: CT, manuscript design and writing; NI, literature searching; HY, contribution as internal medicine consultant.

Conflict of interest: The Authors declare no conflict of interest.

Key words: Tuberculosis; miliary tuberculosis; methotrexate.

Received for publication: 10 April 2019.

Accepted for publication: 8 May 2019.

CCopyright C. Tetikkurt et al., 2019

Licensee PAGEPress, Italy

Monaldi Archives for Chest Disease 2019; 89:1080

doi: 10.4081/monaldi.2019.1080

This article is distributed under the terms of the Creative Commons Attribution Noncommercial License (by-nc 4.0) which permits any noncommercial use, distribution, and reproduction in any medium, provided the original author(s) and source are credited.

\section{Introduction}

Miliary tuberculosis is characterized by the hematogenous dissemination of a large amount of tuberculosis bacilli in the body $[1,2]$. The disease may occur in several organs or throughout the entire body. Miliary tuberculosis leads to a fatal outcome if left untreated or if the treatment is delayed $[3,4]$. Half of the patients are diagnosed antemortem because the infection mimics malignant, inflammatory or reactive diseases [5,6]. Early empirical treatment for possible but not yet definitive miliary tuberculosis is the hallmark to increase the likelihood of survival. A high index of clinical suspicion is required for a punctual diagnosis and improved clinical outcomes.

In our case the preliminary diagnosis was methotrexate reaction, lymphoma, miliary tuberculosis, rheumatoid arthritis associated lung disease and pulmonary infection. We present this patient to remark to the potential difficulties associated with the diagnosis of miliary tuberculosis. Our second aim is to emphasize the potential utility of PET/CT in the diagnosis of miliary tuberculosis where PET/ CT guided biopsy may identify the sample site for early diagnosis. High sensitivity of PET/CT detecting inflammatory lesions appears to be a useful diagnostic modality in miliary tuberculosis.

\section{Case report}

A 63-year-old patient was admitted for loss of appetite, subfebrile fever, swelling of the dorsolateral side of the right hand and dyspnea on exertion for three months. Personal history revealed rheumatoid arthritis treated with methotrexate during the last six months. Physical examination was normal except for the painful soft tissue swelling of the right hand. Fever was $37.8^{\circ} \mathrm{C}$. Blood count was within normal limits. ESR was $48 \mathrm{~mm} / \mathrm{h}$ and CRP was $56 \mathrm{IU} / \mathrm{ml}$. Liver function tests, LDH and alkaline phosphatase were mildly elevated. Electrocardiogram (ECG) showed sinus rhythm of $78 / \mathrm{min}$. Chest radiography and thorax computed tomography (CT) (Figure 1) revealed diffuse micronodules in both lungs. Radiography demonstrated swelling of the right hand (Figure 2). Tuberculin test and anti-histone were negative. PET/CT scan demonstrated diffuse FDG uptake in both lungs, the spleen, the right hand, the mediastinal, the right axillary and the cervical lymph nodes (Figures 3 and 4). MR of the right hand showed erosive changes with widespread osteonecrotic areas in carpal bone surfaces, marked irregularities on joint surfaces and tenosynovitis (Figure 5). Needle aspiration biopsy of the right hand was not diagnostic while tru-cut biopsy showed scarce lymphocytes in the tenosynovial tissue, dystrophic calcification in the degenerated and necrotic connective tissue. Direct smear of 
bronchial lavage was negative for mycobacteria. Histopathologic examination of the axillary lymph node was not diagnostic. Initial bronchial lavage culture was negative for mycobacterium tuberculosis but positive for mycobacterium tuberculosis complex-PCR. Transbronchial biopsy of the lung revealed nonspecific inflammation. PET/CT directed hand tissue and the second bronchial lavage culture performed one week later grew mycobacterium tuberculosis confirming the diagnosis of miliary tuberculosis. Treatment with streptomycin, isoniazid, rifampicin, ethambutol and pyrazinamide was commenced. After the initial two months, the treatment was continued with rifampicin, isoniazid and ethambutol. There was a complete clinical response with improvement of all symptoms within six weeks of anti-tuberculous drug treatment and the patient had an uneventful recovery. The preliminary diagnosis was methotrexate drug reaction, lymphoma, rheumatoid arthritis associated lung disease, miliary tuberculosis and pulmonary infection. Positive tissue culture of the right hand directed by PET/CT was the diagnostic hallmark of miliary tuberculosis in our patient while the positive second bronchial lavage culture and a complete response to anti-tuberculous treatment justified the diagnosis of miliary tuberculosis that was associated with the methotrexate induced immunosuppression.

\section{Discussion}

Miliary tuberculosis is the dissemination of Mycobacterium tuberculosis via hematogenous spread. Classic miliary tuberculosis presents as millet like seeding of bacilli in the lung as evidenced on chest radiography or computed tomography. Miliary tuberculosis is seen in 1-3\% of all tuberculosis cases [1-4]. The infection may occur in an extrapulmonary organ in less than $5 \%$ of the cases, in several organs or throughout the entire body $(>90 \%)$, including the brain. It is characterized by a large number of tuberculous bacilli but diagnosis may easily be missed leading to a fatal outcome if early diagnosis and treatment is not implemented. In addition, miliary tuberculosis may mimic many other diseases inflammatory or malignant in nature. Antemortem diagnosis may be as high as 50\% [5-8]. Miliary tuberculosis is a diagnostic dilemma for the clinician and a high index of clinical suspicion is required while prompt diagnosis with instant treatment is the hallmark for improved clinical outcomes. Early empirical treatment for probable miliary tuberculosis increases the likelihood of survival and should be commenced before the definitive diagnosis becomes available.

The preliminary diagnosis in our case was methotrexate induced drug reaction followed by lymphoma, rheumatoid arthritis associated lung disease, miliary tuberculosis and pulmonary infection. The negative anti-histone result excluded the methotrexate effect. Diffuse FDG uptake in the lungs, the spleen, the axillary and the mediastinal lymph nodes was consistent with methotrexate induced lymphoma that was eliminated by hematologic consultation. Although radiologic appearance suggested miliary tuberculosis infection, previous history of tuberculosis or exposure were not present and the tuberculin test was negative. $\mathrm{PET} / \mathrm{CT}$ in this case demonstrated high FDG uptake in various organs including the lungs, spleen, right hand, axillary and mediastinal lymph nodes that pointed out to hematogenous dissemination of the tubercle bacilli. Growth of mycobacterium tuberculosis from the hand tissue biopsy material directed by PET/CT verified the diagnosis of tuberculosis while other diagnostic markers such as the tuberculin test as well as the initial bronchial lavage and transbronchial biopsy were negative. PET/CT is not indicated in infectious or inflammatory diseases but FDG uptake by granulomatous tissues may be a useful guide for the clinician to obtain pathologic samples for diagnostic evaluation. Use of PET/CT may provide strong evidence for the involvement in various organs in miliary tuberculous patients thereby leading the clinician in the accurate diagnostic pathway for sampling. High sensitivity of $\mathrm{PET} / \mathrm{CT}$ in detecting infectious and inflammatory lesions provides an important diagnostic aid for the diagnosis of tuberculosis. Increased FDG washout in delayed images can identify the inflammatory nature of the lesion whereas malignant lesions usually reveal further accumulation of the tracer. PET/CT guided biopsy may facilitate to determine the biopsy sites where other imaging modalities are unable to identify sites available for sampling in such patients where a diagnostic dilemma arises, which was the case in our patient.

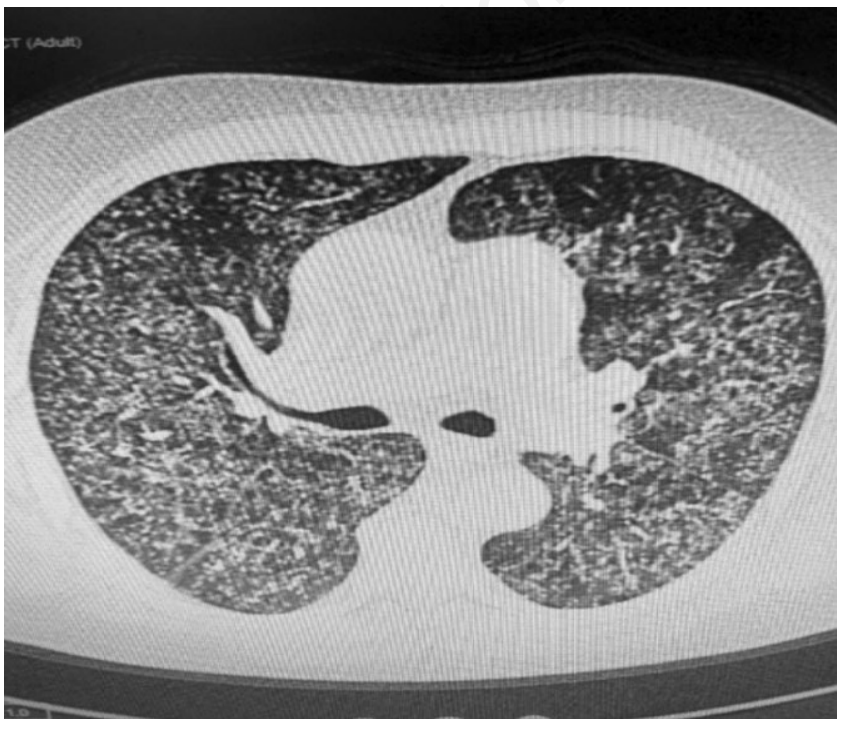

Figure 1. Chest CT revealing diffuse miliary nodules.

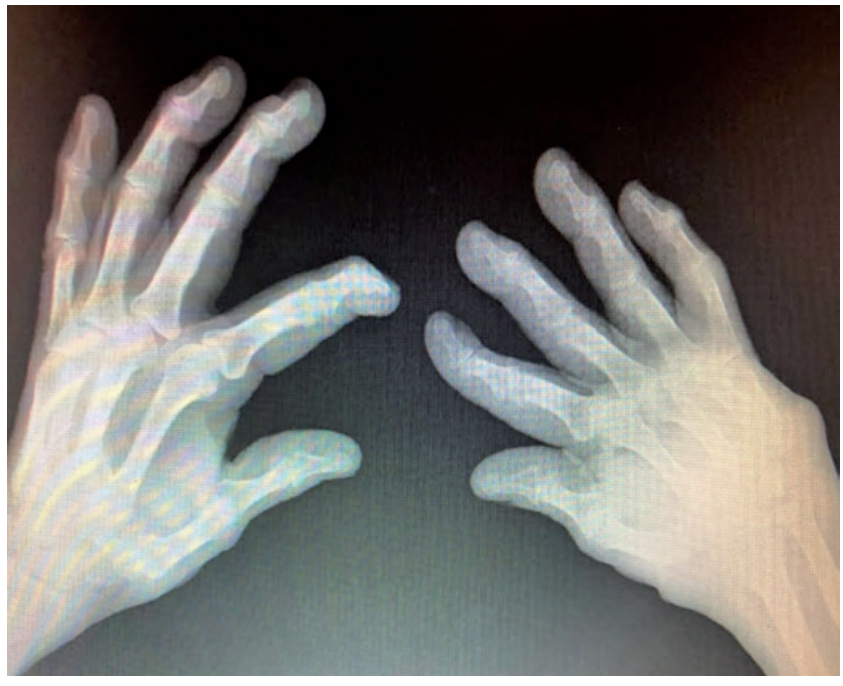

Figure 2. Radiography of the right hand showing soft tissue swelling in the dorsolateral side of the right hand. 
Early treatment for possible but not yet definitive miliary tuberculosis is the hallmark of a successful prognostic outcome. The sooner the treatment is commenced higher the likelihood of achievement and survival. Treatment should never be withheld in any case suspicious for miliary tuberculosis while the test results are pending. On autopsy, multiple tuberculous lesions may be detected throughout the body in organs such as the lungs, liver, spleen, brain and others. There are many studies and case reports relevant to PET/CT utility that reveal the diagnostic and the thera-

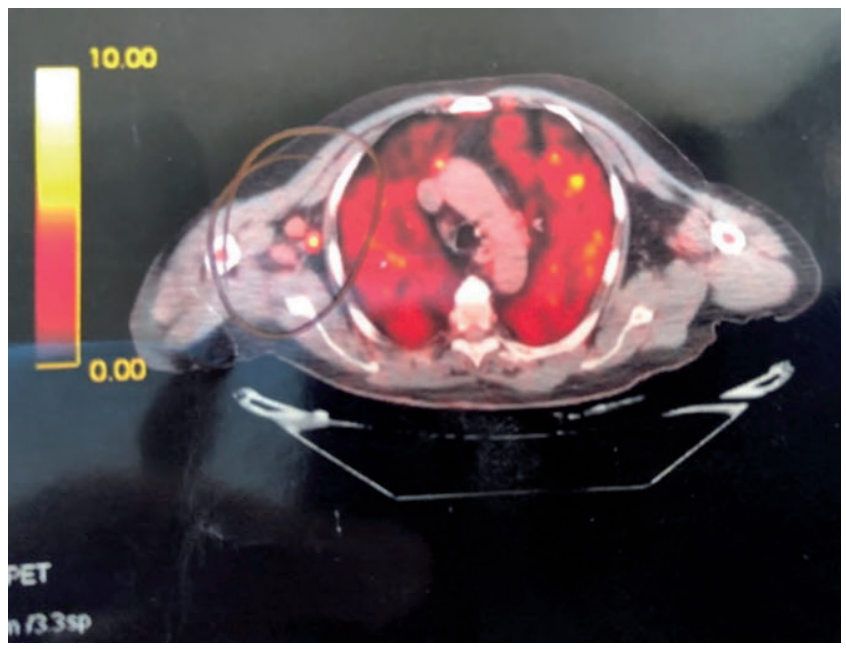

Figure 3. PET/CT showing diffuse FDG uptake in both lungs and in the right axillary lymph node.

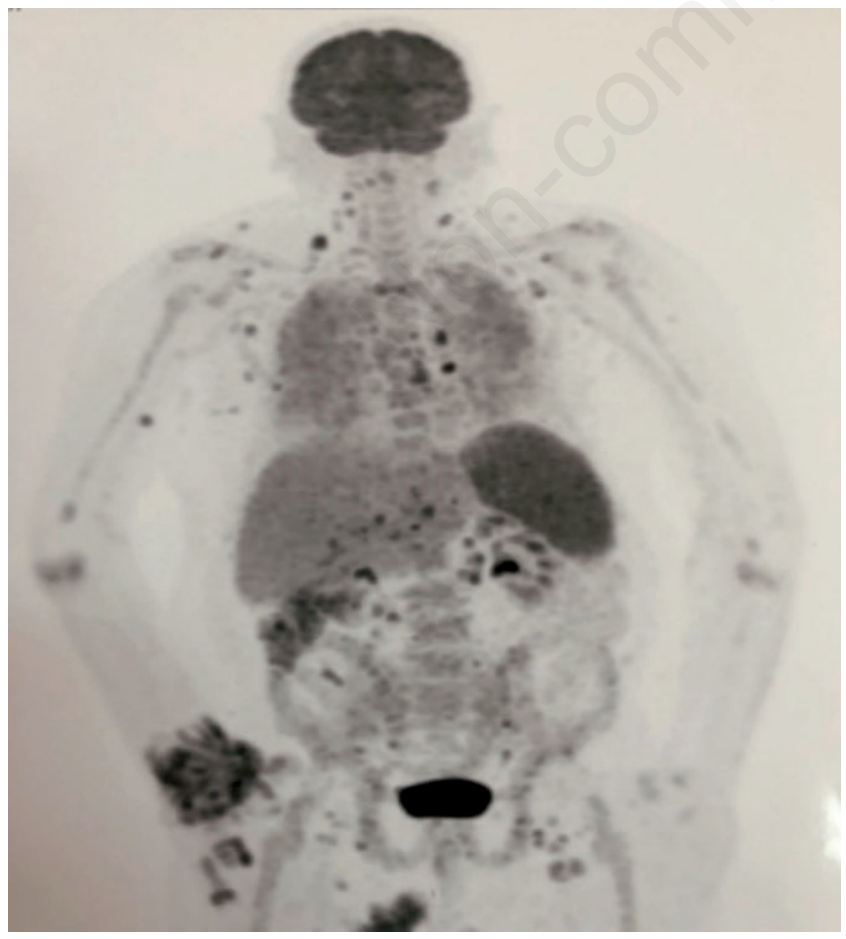

Figure 4. PET/CT revealing high FDG uptake in the lungs, right hand, spleen, axillary, cervical and mediastinal lymph nodes. peutic response evaluation in tuberculosis patients [9-21]. These studies are about the organ involvement of mycobacterium tuberculosis infection while none of these papers have presented data concerning miliary tuberculosis. Our case is unique for miliary tuberculosis revealing increased FDG uptake in various organs indicating the hematogenous dissemination of bacilli which is the hallmark of miliary tuberculosis with identification of the sample site that lead to final diagnosis. In our patient, the culture of the trucut soft tissue hand lesion that was detected by PET/CT established the definitive diagnosis. We conclude that PET/CT may be used as an auxiliary diagnostic tool in miliary tuberculosis that may be extremely useful for locating the sampling sites in cases of negative results from the lung or other involved organs. Detection of the accurate biopsy site by PET/CT may certainly lead the clinician in the orthodox pathway for the final diagnosis in our patient.

Musculoskeletal tuberculosis accounts for approximately $10 \%$ of all extrapulmonary tuberculous cases and is the third most common site after pleural and lymphatic disease. Osteoarticular tuberculosis occurs in $1 \%$ to $3 \%$ of the patients while involvement of the hand is quite rare [22-25]. Tenosynovitis occurs as the most common form of hand tuberculosis. During primary infection bone and synovial tissue may be seeded by bacillemia. Reactivation of tuberculosis arises when immunity is compromised in the setting of HIV, malnutrition, advanced renal disease or during immunosuppressive treatment [26-27]. In our case, the primary mechanism leading to hematogenous dissemination of the tubercle bacilli leading to miliary tuberculosis is the immunosuppression caused by methotrexate treatment used for rheumatoid arthritis. The diagnosis of miliary tuberculosis was confirmed by the positive culture the hand tissue while second bronchial lavage culture positivity was established later in the course of the disease as the first lavage was negative. PET/CT strongly suggested tuberculosis infection and was useful for determining different organs that were associated with hematogenous involvement of miliary tuberculosis. Positive hand tissue culture directed by PET/CT that confirmed the diagnosis of miliary tuberculosis is the first case in literature. PET/CT appears to be a diagnostic facility for early diagnosis and instant treatment that are the hallmarks of a prosperous outcome by identifying sites of active involvement in patients suspicious for miliary tuberculous patients thereby facilitating early sample accommodation. PET/CT guided biopsy may also achieve the diagnosis in cases where locating the sampling site by other imaging modalities have failed. Consequently, PET/CT may yield significant support in cases suspicious for tuberculosis infection by its guidance in obtaining samples for the final diagnosis where early identification and treatment

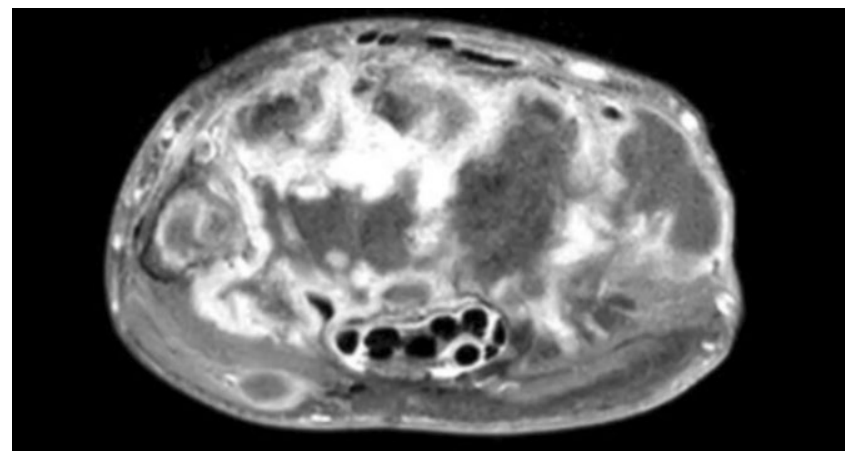

Figure 5. MR of the hand showing erosive changes, widespread osteonecrotic areas in carpal bone surfaces and tenosynovitis. 
are vital for the prognostic outcome of the miliary tuberculosis patients. As far as the cost is considered, PET/CT may increase the financial burden for the patient. On the other hand, use of unsuccessful imaging modalities may produce an equivalent financial load as the PET/CT while causing a delay in diagnosis where early diagnosis and treatment carry a great importance for the outcome of the miliary tuberculosis patient.

\section{Conclusions}

Our findings, symptom screening, sputum smear and mycobacterial culture were limited in their ability to detect tuberculous activity, including latency. Diagnostic laboratory modalities are needed for immediate identification to initiate early treatment of miliary tuberculosis that is the hallmark of a successful prognostic outcome. PET/CT exhibits the wide spread pattern of hematogenous dissemination in miliary tuberculosis that strongly supports the diagnosis. PET/CT may likewise identify the sample site for a tissue culture in the extrapulmonary organs in miliary tuberculosis where the final diagnosis is a dilemma for the clinician and early diagnosis along with instant treatment are of paramount importance for the patient survival. Although the financial burden of PET/CT is high, it should be used as a diagnostic utility in patients with suspected miliary tuberculosis as other failing imaging modalities may be time consuming as well as causing almost an equal expenditure. PET/CT may be more cost and time effective in this kind of patients in regard to other imaging methods.

\section{References}

1. [No authors listed]. Diagnostic Standards and Classification of Tuberculosis in Adults and Children. This official statement of the American Thoracic Society and the Centers for Disease Control and Prevention was adopted by the ATS Board of Directors, July 1999. This statement was endorsed by the Council of the Infectious Disease Society of America, September 1999. Am J Respir Crit Care Med 2000;161:1376-95.

2. Blumberg HM, Burman WJ, Chaisson RE, et al. American Thoracic Society/Centers for Disease Control and Prevention/Infectious Diseases Society of America: treatment of tuberculosis. Am J Respir Crit Care Med 2003;167:603-62.

3. Sharma SK, Mohan A, Sharma A, Mitra DK. Miliary tuberculosis: new insights into an old disease. Lancet Infect Dis 2005;5:415-30.

4. Kim JH, Langston AA, Gallis HA. Miliary tuberculosis: epidemiology, clinical manifestations, diagnosis, and outcome. Rev Infect Dis 1990;12:583-90.

5. Hussain SF, Irfan M, Abbasi M, et al. Clinical characteristics of 110 miliary tuberculosis patients from a low HIV prevalence country. Int J Tuberc Lung Dis 2004;8:493-9.

6. Maartens G, Willcox PA, Benatar SR. Miliary tuberculosis: rapid diagnosis, hematologic abnormalities, and outcome in 109 treated adults. Am J Med 1990;89:291-6.

7. $\mathrm{Mu} \mathrm{XD}$, Wang GF. Images in clinical medicine. Miliary tuberculosis. N Engl J Med 2010;363:1059.
8. Slavin RE, Walsh TJ, Pollack AD. Late generalized tuberculosis: a clinical pathologic analysis and comparison of 100 cases in the preantibiotic and antibiotic eras. Medicine 1980;59:352-66.

9. Martin C, Castaigne C, Vierasu I, et al. Prospective Serial FDG PET/CT During Treatment of Extrapulmonary Tuberculosis in HIV-Infected Patients: An Exploratory Study. Clin Nucl Med 2018;43:635-40.

10. Lang D, Huber H, Kaiser B, et al. SUV as a possible predictor of disease extent and therapy duration in complex tuberculosis. Clin Nucl Med 2018;43:94-100.

11. Ding RL, Cao HY, Hu Y, et al. Lymph node tuberculosis mimicking malignancy on 18F-FDG PET/CT in two patients: A case report. Exp Ther Med 2017;13:3369-73.

12. Stelzmueller I, Huber H, Wunn R, et al. 18F-FDG PET/CT in the Initial Assessment and for Follow-up in Patients With Tuberculosis. Clin Nucl Med 2016;41:187-94.

13. Rayamajhi SJ, Mittal BR, Maturu VN, et al. (18)F-FDG and (18)F-FLT PET/CT imaging in the characterization of mediastinal lymph nodes. Ann Nucl Med 2016;30:207-16.

14. Vorster M, Sathekge MM, Bomanji J. Advances in imaging of tuberculosis: the role of $18 \mathrm{~F}-\mathrm{FDG}$ PET and PET/CT. Curr Opin Pulm Med 2014;20:287-93.

15. Cho YS, Chung DR, Lee EJ et al. 18F-FDG PET/CT in a case of multifocal skeletal tuberculosis without pulmonary disease and potential role for monitoring treatment response. Clin Nucl Med 2014;39:980-3.

16. Bhattacharya A, Kochhar R, Aggrawal K, et al. 18F-FDG and FDG-labeled leukocyte PET/CT in peritoneal tuberculosis. Clin Nucl Med 2014;39:904-5.

17. Dong A, Wang Y, Gong J, Zuo C. FDG PET/CT findings of common bile duct tuberculosis. Clin Nucl Med 2014;39:67-70.

18. Wang JH, Chi CY, Lin KH, et al. Tuberculous arthritisunexpected extrapulmonary tuberculosis detected by FDG PET/CT. Clin Nucl Med 2013;38:93-4.

19. Soussan M, Brillet PY, Mekinian A, et al. Patterns of pulmonary tuberculosis on FDG-PET/CT. Eur J Radiol 2012;81:2872-6.

20. Lee G1, Lee JH, Park SG. F-18 FDG PET/CT imaging of solitary genital tuberculosis mimicking recurrent lymphoma. Clin Nucl Med 2011;36:315-6.

21. Ito K, Morooka M, Kubota K. 18F-FDG PET/CT findings of pharyngeal tuberculosis. Ann Nucl Med 2010;24:493-6.

22. Watts HG, Lifeso RM. Tuberculosis of bones and joints. J Bone Joint Surg Am 1996;78:288-98.

23. Peto HM, Pratt RH, Harrington TA, et al. Epidemiology of extrapulmonary tuberculosis in the United States, 1993-2006. Clin Infect Dis 2009 49:1350-7.

24. Johansen IS, Nielsen SL, Hove $M$ et al. Characteristics and clinical outcome of bone and joint tuberculosis from 1994 to 2011: A retrospective register-based study in Denmark. Clin Infect Dis 2015;61:554-62.

25. Vohra R, Kang HS, Dogra S, et al. Tuberculous osteomyelitis. J Bone Joint Surg Br 1997;79:562-6.

26. Kaufmann SH, Cole ST, Mizrahi V, et al. Mycobacterium tuberculosis and the host response. J Exp Med 2005;201:1693-7.

27. Ellner JJ. Review: the immune response in human tuberculosis--implications for tuberculosis control. J Infect Dis 1997;176:1351-9. 